DOI: $10.17516 / 1997-1397-2020-13-5-547-558$

УДК 517.9

\title{
On Initial Boundary Value Problem for Parabolic Differential Operator with Non-coercive Boundary Conditions
}

\author{
Alexander N. Polkovnikov* \\ Siberian Federal University \\ Krasnoyarsk, Russian Federation
}

Received 10.05.2020, received in revised form 02.06.2020, accepted 20.07.2020

\begin{abstract}
We consider initial boundary value problem for uniformly 2-parabolic differential operator of second order in cylinder domain in $\mathbb{R}^{n}$ with non-coercive boundary conditions. In this case there is a loss of smoothness of the solution in Sobolev type spaces compared with the coercive situation. Using by Faedo-Galerkin method we prove that problem has unique solution in special Bochner space.
\end{abstract}

Keywords: non-coercive problem, parabolic problem, Faedo-Galerkin method.

Citation: A. N. Polkovnikov, On Initial Boundary Value Problem for Parabolic Differential Operator with Non-coercive Boundary Conditions, J. Sib. Fed. Univ. Math. Phys., 2020, 13(5), 547-558.

DOI: $10.17516 / 1997-1397-2020-13-5-547-558$.

Initial boundary value problems for parabolic (by Petrovsky) differential operators with coercive boundary conditions are well studied (see, for instance, [1-4]). However the problem with non-coercive boubdary conditions are also appeared in both theory and applications, see, for instance, pioneer work in this direction [5] and papers $[6,7]$ and $[8]$ for such problems in the Elasticity Theory. Recent results in Fredholm operator equations, induced by boundary value problems for elliptic differential operators with non-coercive boundary conditions (see, for instance, [9-12]) allows us to apply these one for studying the parabolic problem. Consideration of such problems essentially extends variety of boundary operators, but there is a loss of regularity of the solution (see [13] for elliptic case). Namely, let $\Omega_{T}$ be a cylinder,

$$
\Omega_{T}=\Omega \times(0, T),
$$

where $\Omega$ is a bounded domain in $\mathbb{R}^{n}$.

Consider a second order differential operator

$$
A(x, t, \partial)=-\sum_{i, j=1}^{n} \partial_{i}\left(a_{i, j}(x) \partial_{j} \cdot\right)+\sum_{j=1}^{n} a_{j}(x) \partial_{l}+a_{0}(x)+\frac{\partial}{\partial t}
$$

of divergence form in the domain $\Omega_{T}$. The coefficients $a_{i, j}, a_{j}$ are assumed to be complex-valued functions of class $L^{\infty}(\Omega)$. We suppose that the matrix $\mathfrak{A}(x)=\left(a_{i, j}(x)\right)_{\substack{i=1, \ldots, n \\ j=1, \ldots, n}}$ is Hermitian and satisfies

$$
\sum_{i, j=1}^{n} a_{i, j}(x) \bar{w}_{i} w_{j} \geqslant 0 \text { for all }(x, w) \in \bar{\Omega} \times \mathbb{C}^{n},
$$

*paskaattt@yandex.ru https://orcid.org/0000-0002-7066-3219.

(C) Siberian Federal University. All rights reserved 


$$
\sum_{i, j=1}^{n} a_{i, j}(x) \xi_{i} \xi_{j} \geqslant m|\xi|^{2} \text { for all }(x, \xi) \in \bar{\Omega} \times\left(\mathbb{R}^{n} \backslash\{0\}\right),
$$

where $m$ is a positive constant independent of $x$ and $\xi$. Estimate (2) is nothing but the statement that the operator $A(x, t, \partial)$ is uniformly 2-parabolic.

We note that, since the coefficients of the operator and the functions under consideration are complex-valued, inequalities (1) and (2) are weaker than

$$
\sum_{i, j=1}^{n} a_{i, j}(x) \bar{w}_{i} w_{j} \geqslant m|w|^{2}
$$

for all $(x, w) \in \bar{\Omega} \times\left(\mathbb{C}^{n} \backslash\{0\}\right)$. Inequality (3) means that correspondent Hermitian form (see form (4)) is coercive.

Consider boundary operator of Robin type:

$$
B(x, \partial)=b_{1}(x) \sum_{i, j=1}^{n} a_{i, j}(x) \nu_{i} \partial_{j}+b_{0}(x),
$$

where $b_{0}, b_{1}$ are bounded functions on $\partial \Omega$ and $\nu(x)=\left(\nu_{1}(x), \ldots, \nu_{n}(x)\right)$ is the unit outward normal vector of $\partial \Omega$ at $x \in \partial \Omega$. Let $S$ be an open connected subset of $\partial \Omega$ with piecewise smooth boundary $\partial S$. We allow the function $b_{1}(x)$ to vanish on $S$. In this case we assume that $b_{0}(x)$ does not vanish for $x \in S$.

Consider now the following mixed initial-boundary problem in a bounded domain $\Omega_{T}$ with Lipschitz boundary $\partial \Omega_{T}$.

Problem 1. Find a distribution $u(x, t)$, satisfying the problem

$$
\left\{\begin{array}{ccccc}
A(x, t, \partial) u & = & f & \text { in } & \Omega_{T} \\
B(x, \partial) u & = & 0 & \text { on } & \partial \Omega \times(0, T) \\
u(x, 0) & = & u_{0} & \text { on } & \Omega
\end{array}\right.
$$

with given data $f \in \Omega_{T}$.

For solving the problem we have to define appropriate functional spaces. Denote by $C^{1}(\bar{\Omega}, S)$ the subspace of $C^{1}(\bar{\Omega})$ consisting of those functions whose restriction to the boundary vanishes on $\bar{S}$. Let $H^{1}(\Omega, S)$ be the closure of $C^{1}(\bar{\Omega}, S)$ in $H^{1}(\Omega)$. Since on $S$ the boundary operator reduces to $B=b_{0}(x)$ and $b_{0}(x) \neq 0$ for $x \in S$, then the functions $u \in H^{1}(\Omega)$ satisfying $B u=0$ on $\partial \Omega$ belong to $H^{1}(\Omega, S)$.

Split now both $a_{0}(x)$ and $b_{0}(x)$ into two parts

$$
\begin{gathered}
a_{0}=a_{0,0}+\delta a_{0}, \\
b_{0}=b_{0,0}+\delta b_{0},
\end{gathered}
$$

where $a_{0,0}$ is a non-negative bounded function in $\Omega$ and $b_{0,0}$ is a such function that $b_{0,0} / b_{1}$ is non-negative bounded function on $S$. Then, under reasonable assumptions, the Hermitian form

$$
(u, v)_{+}=\int_{\Omega} \sum_{i, j=1}^{n} a_{i, j} \partial_{j} u \overline{\partial_{i} v} d x+\left(a_{0,0} u, v\right)_{L^{2}(\Omega)}+\left(b_{0,0} / b_{1} u, v\right)_{L^{2}(\partial \Omega \backslash S)}
$$

defines the scalar product on $H^{1}(\Omega, S)$. Denote by $H^{+}(\Omega)$ the completion of the space $H^{1}(\Omega, S)$ with respect to the corresponding norm $\|\cdot\|_{+}$. From now on we assume that the space $H^{+}(\Omega)$ is 
continuously embedded into the Lebesgue space $L^{2}(\Omega)$, i.e. there is a constant $c>0$, independent of $u$, such that

$$
\|u\|_{L^{2}(\Omega)} \leqslant c\|u\|_{+} \text {for all } u \in H^{+}(\Omega) .
$$

It is true, if there exist a positive constant $c_{1}$ such that

$$
a_{0,0} \geqslant c_{1} \text { in } \Omega \text {. }
$$

Actually we can get more subtle embedding for the space $H^{+}(\Omega)$.

Theorem 2. Let the coefficients $a_{i, j}$ be $C^{\infty}$ in a neighbourhood of the closure of $\Omega$, inequalities (1), (2) hold and

$$
\frac{b_{0,0}}{b_{1}} \geqslant c_{2} \text { at } \partial \Omega \backslash S,
$$

with some constant $c_{2}>0$. Then the space $H^{+}(\Omega)$ is continuously embedded into $H^{1 / 2-\varepsilon}(\Omega)$ for any $\varepsilon>0$ if there is a positive constant $c_{1}$, such that

$$
a_{0,0} \geqslant c_{1} \text { in } \Omega
$$

or the operator $A$ is strongly elliptic in a neighborhood $X$ of $\bar{\Omega}$ and

$$
\int_{X} \sum_{i, j=1}^{n} a_{i, j} \partial_{j} u \overline{\partial_{i} u} d x \geqslant m\|u\|_{L^{2}(X)}^{2}
$$

for all $u \in C_{\text {comp }}^{\infty}(X)$, with $m>0$ a constant independent of $u$.

Proof. See [12, Theorem 2.5].

Of course, under coercive estimate (3), the space $H^{+}(\Omega)$ is continuously embedded into $H^{1}(\Omega)$. However, in general, the embedding, described in Theorem 2 is rather sharp (see [12, Remark 5.1]).

The absence of coerciveness does not allows to consider arbitrary derivatives $\partial_{j} u$ for an element $u \in H^{+}(\Omega)$. To cope with this difficulty we note that the matrix $\mathfrak{A}(x)=\left(a_{i, j}(x)\right)_{\substack{i=1, \ldots, n \\ j=1, \ldots, n}}$ admits a factorisation, i.e. there is an $(m \times n)$-matrix $\mathfrak{D}(x)=\left(\mathfrak{D}_{i, j}(x)\right)_{\substack{i=1, \ldots, m \\ j=1, \ldots, n}}$ of bounded functions in $\Omega$, such that

$$
(\mathfrak{D}(x))^{*} \mathfrak{D}(x)=\mathfrak{A}(x)
$$

for almost all $x \in D$ (see, for instance, [14]). For example, one could take the standard nonnegative self-adjoint square root $\mathfrak{D}(x)=\sqrt{\mathfrak{A}(x)}$ of the matrix $\mathfrak{A}(x)$. Then

$$
\sum_{i, j=1}^{n} a_{i, j} \partial_{j} u \overline{\partial_{i} v}=(\mathfrak{D} \nabla v)^{*} \mathfrak{D} \nabla u=\sum_{l=1}^{m} \overline{\mathfrak{D}_{l} v} \mathfrak{D}_{l} u,
$$

for all smooth functions $u$ and $v$ in $\Omega$, where $\nabla u$ is thought of as $n$-column with entries $\partial_{1} u, \ldots, \partial_{n} u$, and $\mathfrak{D}_{l} u:=\sum_{s=1}^{n} \mathfrak{D}_{l, s}(x) \partial_{s} u, l=1, \ldots, m$. From now on we may confine ourselves with first order summand of the form

$$
\sum_{l=1}^{m} \tilde{a}_{l}(x) \mathfrak{D}_{l}, \quad \tilde{a}_{l}(x) \in L^{\infty}(\Omega)
$$


instead of

$$
\sum_{j=1}^{n} a_{j}(x) \partial_{j}
$$

Since the coefficients $\delta a_{0}, \tilde{a}_{l}$ belong to $L^{\infty}(\Omega)$ for all $l=0, \ldots, m$, it follows from Cauchy inequality that

$$
\left|\left(\left(\sum_{l=1}^{m} \tilde{a}_{l}(x) \mathfrak{D}_{l}+\delta a_{0}\right) u, v\right)_{L^{2}(\Omega)}\right| \leqslant c\|u\|_{+}\|v\|_{+} .
$$

Let now $H^{-}(\Omega)$ stand for the dual space for the space $H^{+}(\Omega)$ with respect to the pairing $<\cdot, \cdot\rangle$ induced by the scalar product $(\cdot, \cdot)_{L^{2}(\Omega)}$, see $[2,15]$ and elsewhere. It is a Banach space with the norm

$$
\|u\|_{-}=\sup _{\substack{v \in H^{+}(\Omega) \\ v \neq 0}} \frac{\left|(v, u)_{L^{2}(\Omega)}\right|}{\|v\|_{+}} .
$$

The space $L^{2}(\Omega)$ is continuously embedded into $H^{-}(\Omega)$, if the space $H^{+}(\Omega)$ is continuously embedded into $L^{2}(\Omega)$ (see [9]). We denote by $i^{\prime}: L^{2}(\Omega) \rightarrow H^{-}(\Omega)$ and $i: H^{+}(\Omega) \rightarrow L^{2}(\Omega)$ the operators of correspondent continuously embeddings. Thus we have a triple of the functional spaces

$$
H^{+}(\Omega) \stackrel{i}{\hookrightarrow} L^{2}(\Omega) \stackrel{i^{\prime}}{\hookrightarrow} H^{-}(\Omega),
$$

where each embeddings is compact under the hypothesis of Theorem 2.

Denote by $L^{2}\left(0, T ; H^{+}(\Omega)\right)$ the Bochner space of $L^{2}$-functions

$$
u(t):[0, T] \rightarrow H^{+}(\Omega) .
$$

It is a Banach space with the norm

$$
\|u\|_{L^{2}\left(0, T ; H^{+}(\Omega)\right)}^{2}=\int_{0}^{T}\|u(t)\|_{+}^{2} d t .
$$

Then an integration by parts in $\Omega$ leads to a weak formulation of Problem (1):

Problem 3. Given $f \in L^{2}\left(0, T ; H^{-}(\Omega)\right)$ and $u_{0} \in L^{2}(\Omega)$, find $u \in L^{2}\left(0, T ; H^{+}(\Omega)\right)$, such that

$$
(u, v)_{+}+\left(\left(\sum_{l=1}^{m} \tilde{a}_{l}(x) \mathfrak{D}_{l}+\delta a_{0}\right) u, v\right)_{L^{2}(\Omega)}+\frac{\partial}{\partial t}(u, v)_{L^{2}(\Omega)}=<f, v>
$$

for all $v \in H^{+}(\Omega)$, and

$$
u(0)=u_{0} .
$$

In general case the condition (11) have no sense for functions $u \in L^{2}\left(0, T ; H^{+}(\Omega)\right)$. But we will see below that function $u(t) \in L^{2}\left(0, T ; H^{+}(\Omega)\right)$, satisfying (10), is continuous and (11) have a sense.

We want to apply the Faedo-Galerkin method for solving the Problem 3 (see, for instance, $[2,4])$. For this purpose we need some complete system of vectors in the space $H^{+}(\Omega)$. As this system we take the set of eigenvectors of an operator, induced by the weak statement of elliptic selfadjoint problem, corresponding to the parabolic Problem 3. Namely, for given $f \in H^{-}(\Omega)$, find $u \in H^{+}(\Omega)$, such that

$$
(u, v)_{+}+\left(\left(\sum_{l=1}^{m} \tilde{a}_{l}(x) \mathfrak{D}_{l}+\delta a_{0}\right) u, v\right)_{L^{2}(\Omega)}=<f, v>.
$$


Equality (12) induces a bounded linear operator $L: H^{+}(\Omega) \rightarrow H^{-}(\Omega)$,

$$
(u, v)_{+}+\left(\left(\sum_{l=1}^{m} \tilde{a}_{l}(x) \mathfrak{D}_{l}+\delta a_{0}\right) u, v\right)_{L^{2}(\Omega)}=<L u, v>
$$

Denote by $L_{0}$ the operator $L$ in the case, when $\delta a_{0}=a_{l}=0$ for all $l=1, \ldots, m$,

$$
(u, v)_{+}=<L_{0} u, v>
$$

The operator $L_{0}: H^{+}(\Omega) \rightarrow H^{-}(\Omega)$ is continuously invertible and $\left\|L_{0}\right\|=\left\|L_{0}^{-1}\right\|=1$ (see [12, Lemma 2.6]). According to [12, Lemma 3.1], there is a system $\left\{h_{j}\right\}$ of eigenvectors of the compact positive selfadjoint operator $L_{0}^{-1} i^{\prime} i: H^{+}(\Omega) \rightarrow H^{+}(\Omega)$, which is an orthonormal bases in $H^{+}(\Omega)$ and an orthogonal bases in $L^{2}(\Omega)$ and $H^{-}(\Omega)$.

Let now function $u \in L^{2}\left(0, T ; H^{+}(\Omega)\right)$ satisfies (10). We have from (13)

$$
\left(\frac{\partial u}{\partial t}, v\right)_{L^{2}(\Omega)}=<\frac{\partial u}{\partial t}, v>=<f-L u, v>
$$

Since $f \in L^{2}\left(0, T ; H^{-}(\Omega)\right)$ and operator $L: H^{+}(\Omega) \rightarrow H^{-}(\Omega)$ is bounded, then $\frac{\partial u}{\partial t} \in$ $L^{2}\left(0, T ; H^{-}(\Omega)\right)$. It means, that

$$
u \in C\left(0, T ; L^{2}(\Omega)\right)
$$

(see, for instance, [2] or [16]).

Using by the standard Faedo-Galerkin method (see, for instance, $[1,2,4]$ ) we get next Theorem.

Theorem 4. Under the hypothesis of Theorem 2, the Problem 3 has at least one solution $u(t)$, and, moreover, $u(t) \in C\left(0, T ; L^{2}(\Omega)\right)$.

Proof. For each k we are looking for approximate solution of Problem 3 on the next form

$$
u_{k}(t)=\sum_{j=1}^{k} g_{j k}(t) h_{j}
$$

and function $u_{k}$ satisfies

$$
\begin{gathered}
\left(u_{k}, h_{i}\right)_{+}+\left(\left(\sum_{l=1}^{m} \tilde{a}_{l}(x) \mathfrak{D}_{l}+\delta a_{0}\right) u_{k}, h_{i}\right)_{L^{2}(\Omega)}+\left(\frac{\partial u_{k}}{\partial t}, h_{i}\right)_{L^{2}(\Omega)}=<f, h_{i}> \\
u_{k}(0)=\sum_{j=1}^{k} \frac{\left(u_{0}, h_{j}\right)_{L^{2}(\Omega)}}{\left\|h_{j}\right\|_{L^{2}(\Omega)}^{2}} h_{j}
\end{gathered}
$$

for each $j=1, \ldots, k$, where $\left\{h_{j}\right\}$ is the orthonormal bases in $H^{+}(\Omega)$. It means that $(17)$ takes the form

$$
g_{i k}(t)+\sum_{j=1}^{k}\left(\left(\sum_{l=1}^{m} \tilde{a}_{l}(x) \mathfrak{D}_{l}+\delta a_{0}\right) h_{j}, h_{i}\right)_{L^{2}(\Omega)} g_{j k}(t)+g_{i k}^{\prime}(t)\left\|h_{i}\right\|_{L^{2}(\Omega)}^{2}=<f, h_{i}>,
$$

where $i=1, \ldots, k$. It is a system of linear differential equations of first order with initial conditions

$$
g_{i k}(0)=\frac{\left(u_{0}, h_{i}\right)_{L^{2}(\Omega)}}{\left\|h_{i}\right\|_{L^{2}(\Omega)}^{2}}, \quad i=1, \ldots, k .
$$


Since $<f(t), h_{i}>$ is measurable function for all $i=1, \ldots, k$, then there is unique function $g_{i k}(t)$ for each $i=1, \ldots, k$, satisfying (19) and (20) for all $t \in[0, T]$ (see, for instance, [17]). Note, as the function $u(t)$ is complex-valued, then the functions $\left\{g_{i k}(t)\right\}$ may be complex-valued too and the system (19) consists $2 k$ real-valued equations in general case.

Now we have to get some priori estimates for function $u_{k}(t)$ independent of $k$. Multiplying the equality (17) by the $\overline{g_{i k}(t)}$ and summing by $i=1, \ldots, k$ we get

$$
\left\|u_{k}\right\|_{+}^{2}+\left(\frac{\partial u_{k}}{\partial t}, u_{k}\right)_{L^{2}(\Omega)}=<f, u_{k}>-\left(\left(\sum_{l=1}^{m} \tilde{a}_{l}(x) \mathfrak{D}_{l}+\delta a_{0}\right) u_{k}, u_{k}\right)_{L^{2}(\Omega)} .
$$

Hence, by the Cauchy inequality,

$$
\begin{aligned}
& 2\left|\left\|u_{k}\right\|_{+}^{2}+\left(\frac{\partial u_{k}}{\partial t}, u_{k}\right)_{L^{2}(\Omega)}\right|= \\
= & 2\left|<f, u_{k}>-\left(\sum_{l=1}^{m} \tilde{a}_{l}(x) \mathfrak{D}_{l} u_{k}, u_{k}\right)_{L^{2}(\Omega)}-\left(\delta a_{0} u_{k}, u_{k}\right)_{L^{2}(\Omega)}\right| \leqslant \\
\leqslant & \|f\|_{-}^{2}+\left\|u_{k}\right\|_{+}^{2}+2 c_{1}\left\|u_{k}\right\|_{+}\left\|u_{k}\right\|_{L^{2}(\Omega)}+2 c_{2}\left\|u_{k}\right\|_{L^{2}(\Omega)}^{2} \leqslant \\
\leqslant & \|f\|_{-}^{2}+\frac{3}{2}\left\|u_{k}\right\|_{+}^{2}+\left(2 c_{2}+2 c_{1}^{2}\right)\left\|u_{k}\right\|_{L^{2}(\Omega)}^{2}
\end{aligned}
$$

for some positive constants $c_{1}$ and $c_{2}$. As the norm $\left\|u_{k}\right\|_{+}^{2}$ is a real-valued function, we have

$$
\begin{aligned}
& 2\left|\left\|u_{k}\right\|_{+}^{2}+\left(\frac{\partial u_{k}}{\partial t}, u_{k}\right)_{L^{2}(\Omega)}\right|= \\
& =2\left|\left\|u_{k}\right\|_{+}^{2}+\mathfrak{R e}\left(\left(\frac{\partial u_{k}}{\partial t}, u_{k}\right)_{L^{2}(\Omega)}\right)+i \mathfrak{I m}\left(\left(\frac{\partial u_{k}}{\partial t}, u_{k}\right)_{L^{2}(\Omega)}\right)\right| \geqslant \\
& \geqslant 2\left\|u_{k}\right\|_{+}^{2}+2 \mathfrak{R e}\left(\left(\frac{\partial u_{k}}{\partial t}, u_{k}\right)_{L^{2}(\Omega)}\right),
\end{aligned}
$$

where $\mathfrak{R} \mathfrak{e}(g)$ and $\mathfrak{I m}(g)$ denote real and imaginary parts of function $g$ respectively. On the other hand,

$$
\begin{aligned}
\frac{d}{d t}\left\|u_{k}\right\|_{L^{2}(\Omega)}^{2} & =\left(\frac{\partial u_{k}}{\partial t}, u_{k}\right)_{L^{2}(\Omega)}+\left(u_{k}, \frac{\partial u_{k}}{\partial t}\right)_{L^{2}(\Omega)}= \\
& =2 \mathfrak{R e}\left(\left(\frac{\partial u_{k}}{\partial t}, u_{k}\right)_{L^{2}(\Omega)}\right) .
\end{aligned}
$$

It follows from (22), (23) and (24) that

$$
\frac{1}{2}\left\|u_{k}(t)\right\|_{+}^{2}+\frac{d}{d t}\left\|u_{k}(t)\right\|_{L^{2}(\Omega)}^{2} \leqslant\|f(t)\|_{-}^{2}+\left(2 c_{2}+2 c_{1}^{2}\right)\left\|u_{k}\right\|_{L^{2}(\Omega)}^{2} .
$$

Now, integrating (25) by $t$ from 0 till some $s \in(0, T)$ we get

$$
\begin{gathered}
\frac{1}{2} \int_{0}^{s}\left\|u_{k}(t)\right\|_{+}^{2} d t+\left\|u_{k}(s)\right\|_{L^{2}(\Omega)}^{2}-\left\|u_{k}(0)\right\|_{L^{2}(\Omega)}^{2} \leqslant \\
\leqslant \int_{0}^{s}\|f(t)\|_{-}^{2} d t+\left(2 c_{2}+2 c_{1}^{2}\right) \int_{0}^{s}\left\|u_{k}\right\|_{L^{2}(\Omega)}^{2} d t .
\end{gathered}
$$


Since the sequence $\left\{u_{k}(0)\right\}$ seeks to $u_{0}$ with $k \rightarrow \infty$ strongly in $L^{2}(\Omega)$, it follows from Gronwall type lemma (see [18] or [19]), that

$$
\left\|u_{k}(s)\right\|_{L^{2}(\Omega)}^{2} \leqslant\left(\left\|u_{0}\right\|_{L^{2}(\Omega)}^{2}+\int_{0}^{T}\|f(t)\|_{-}^{2} d t\right) e^{\left(2 c_{2}+2 c_{1}^{2}\right) s} .
$$

Hence

$$
\sup _{s \in[0, T]}\left\|u_{k}(s)\right\|_{L^{2}(\Omega)}^{2} \leqslant\left(\left\|u_{0}\right\|_{L^{2}(\Omega)}^{2}+\int_{0}^{T}\|f(t)\|_{-}^{2} d t\right) e^{\left(2 c_{2}+2 c_{1}^{2}\right) T} .
$$

The right side of (26) independent of $k$, therefore the sequence $\left\{u_{k}(t)\right\}$ is bounded in $L^{\infty}\left(0, T ; L^{2}(\Omega)\right)$. Then there is a subsequence $\left\{u_{k^{\prime}}(t)\right\}$ of the sequence $\left\{u_{k}(t)\right\}$ and an element $u(t) \in L^{\infty}\left(0, T ; L^{2}(\Omega)\right)$ such that $u_{k^{\prime}}(t) \rightarrow u(t)$ in the weak-* topology of $L^{\infty}\left(0, T ; L^{2}(\Omega)\right)$, namely

$$
\lim _{k^{\prime} \rightarrow \infty} \int_{0}^{T}\left(u_{k^{\prime}}(t)-u(t), v(t)\right)_{L^{2}(\Omega)} d t=0
$$

for all $v \in L^{1}\left(0, T ; L^{2}(\Omega)\right)$.

Integrating again (25) by $t$ from 0 till $T$ and applying Gronwall type lemma we have

$$
\begin{aligned}
& \frac{1}{2} \int_{0}^{T}\left\|u_{k}(t)\right\|_{+}^{2} d t+\left\|u_{k}(T)\right\|_{L^{2}(\Omega)}^{2} \leqslant \\
& \leqslant\left(\left\|u_{0}\right\|_{L^{2}(\Omega)}^{2}+\int_{0}^{T}\|f(t)\|_{-}^{2} d t\right) e^{\left(2 c_{2}+2 c_{1}^{2}\right) T} .
\end{aligned}
$$

It means that the sequence $\left\{u_{k}(t)\right\}$ is bounded in $L^{2}\left(0, T ; H^{+}(\Omega)\right)$. In particular, the sequence $\left\{u_{k^{\prime}}(t)\right\}$ is bounded in $L^{2}\left(0, T ; H^{+}(\Omega)\right)$ too. Hence there is a subsequence $\left\{u_{k^{\prime \prime}}(t)\right\}$ of the sequence $\left\{u_{k^{\prime}}(t)\right\}$ and an element $\widetilde{u}(t) \in L^{2}\left(0, T ; H^{+}(\Omega)\right)$ such that $u_{k^{\prime \prime}}(t) \rightarrow u(t)$ in the weak topology of $L^{2}\left(0, T ; H^{+}(\Omega)\right)$,

$$
\lim _{k^{\prime \prime} \rightarrow \infty} \int_{0}^{T}\left(u_{k^{\prime \prime}}(t), v\right)_{+} d t=\int_{0}^{T}(u(t), v)_{+} d t
$$

for all $v \in L^{2}\left(0, T ; H^{+}(\Omega)\right)$ and

$$
\lim _{k^{\prime \prime} \rightarrow \infty} \int_{0}^{T}<u_{k^{\prime \prime}}(t)-\widetilde{u}(t), v(t)>d t=0
$$

for all $v \in L^{2}\left(0, T ; H^{-}(\Omega)\right)$. In particular

$$
\lim _{k^{\prime \prime} \rightarrow \infty} \int_{0}^{T}\left(u_{k^{\prime \prime}}(t), v(t)\right)_{L^{2}(\Omega)} d t=\int_{0}^{T}(\widetilde{u}(t), v(t))_{L^{2}(\Omega)} d t
$$

for all $v \in L^{2}\left(0, T ; L^{2}(\Omega)\right)$.

From (27) and (31) we have

$$
\int_{0}^{T}(u(t)-\widetilde{u}(t), v(t))_{L^{2}(\Omega)} d t=0
$$

for all $v \in L^{2}\left(0, T ; L^{2}(\Omega)\right)$. Hence

$$
u(t)=\widetilde{u}(t) \in L^{\infty}\left(0, T ; L^{2}(\Omega)\right) \cap L^{2}\left(0, T ; H^{+}(\Omega)\right) .
$$


From now on we denote by $\left\{u_{k}(t)\right\}$ the subsequence $\left\{u_{k^{\prime \prime}}(t)\right\}$.

Let now $\psi(t)$ be a scalar differentiable function on $[0, T]$ such that $\psi(T)=0$. Multiplying (17) by $\psi(t)$ and integrating by $t$ we get

$$
\begin{aligned}
\int_{0}^{T}\left(u_{k}(t), h_{j}\right)_{+} \psi(t) d t+ & \int_{0}^{T}\left(\left(\sum_{l=1}^{m} \tilde{a}_{l}(x) \mathfrak{D}_{l}+\delta a_{0}\right) u_{k}(t), h_{i}\right)_{L^{2}(\Omega)} \psi(t) d t+ \\
& +\int_{0}^{T}\left(\frac{\partial u_{k}(t)}{\partial t}, h_{i}\right)_{L^{2}(\Omega)} \psi(t) d t=\int_{0}^{T}<f(t), h_{j}>\psi(t) d t .
\end{aligned}
$$

However

$$
\int_{0}^{T}\left(\frac{\partial u_{k}(t)}{\partial t}, h_{i}\right)_{L^{2}(\Omega)} \psi(t) d t=-\int_{0}^{T}\left(u_{k}(t), \psi^{\prime}(t) h_{j}\right)_{L^{2}(\Omega)} d t-\left(u_{k}(0), h_{j} \psi(0)\right)_{L^{2}(\Omega)}
$$

and it follows that

$$
\begin{aligned}
& \int_{0}^{T}\left(u_{k}(t), h_{j} \psi(t)\right)_{+} d t+\int_{0}^{T}\left(\left(\sum_{l=1}^{m} \tilde{a}_{l}(x) \mathfrak{D}_{l}+\delta a_{0}\right) u_{k}(t), h_{i}\right)_{L^{2}(\Omega)} \psi(t) d t- \\
& \quad-\int_{0}^{T}\left(u_{k}(t), \psi^{\prime}(t) h_{j}\right)_{L^{2}(\Omega)} d t=\left(u_{k}(0), h_{j} \psi(0)\right)_{L^{2}(\Omega)}+\int_{0}^{T}<f(t), h_{j}>\psi(t) d t .
\end{aligned}
$$

Now we want to go to the limit in (36) with $k \rightarrow \infty$. It follows from 9 , that

$$
\int_{0}^{T}\left(\left(\sum_{l=1}^{m} \tilde{a}_{l}(x) \mathfrak{D}_{l}+\delta a_{0}\right) u_{k}(t), h_{i}\right)_{L^{2}(\Omega)} \psi(t) d t
$$

is continuous linear functional on $L^{2}\left(0, T ; H^{+}(\Omega)\right)$. Since $u_{k}(t) \rightarrow u(t)$ with $k \rightarrow \infty$ in the weak topology of $L^{2}\left(0, T ; H^{+}(\Omega)\right)$, we have

$$
\lim _{k \rightarrow \infty} \int_{0}^{T}\left(\left(\sum_{l=1}^{m} \tilde{a}_{l}(x) \mathfrak{D}_{l}+\delta a_{0}\right)\left(u_{k}(t)-u(t)\right), h_{i}\right)_{L^{2}(\Omega)} \psi(t) d t=0
$$

From (31), (29), (33) and the fact that $u_{k}(0) \rightarrow u_{0}$ strongly in $L^{2}(\Omega)$ with $k \rightarrow \infty$ we get

$$
\begin{aligned}
\int_{0}^{T}\left(u(t), h_{j} \psi(t)\right)_{+} d t+\int_{0}^{T}\left(\left(\sum_{l=1}^{m} \tilde{a}_{l}(x) \mathfrak{D}_{l}+\delta a_{0}\right) u(t), h_{i} \psi(t)\right)_{L^{2}(\Omega)} d t- \\
\quad-\int_{0}^{T}\left(u(t), \psi^{\prime}(t) h_{j}\right)_{L^{2}(\Omega)} d t=\left(u_{0}, h_{j} \psi(0)\right)_{L^{2}(\Omega)}+\int_{0}^{T}<f(t), h_{j}>\psi(t) d t
\end{aligned}
$$

As the system $\left\{h_{j}\right\}_{j=1,2, \ldots}$ is dense in $H^{+}(\Omega)$ and $L^{2}(\Omega)$, equality (37) holds by linearity and continuity for all $v \in H^{+}(\Omega)$,

$$
\begin{aligned}
\int_{0}^{T}(u(t), v)_{+} \psi(t) d t+\int_{0}^{T}\left(\left(\sum_{l=1}^{m} \tilde{a}_{l}(x) \mathfrak{D}_{l}+\delta a_{0}\right) u(t), v\right)_{L^{2}(\Omega)} \psi(t) d t- \\
-\int_{0}^{T}(u(t), v)_{L^{2}(\Omega)} \psi^{\prime}(t) d t=\left(u_{0}, v\right)_{L^{2}(\Omega)} \psi(0)+\int_{0}^{T}<f(t), v>\psi(t) d t .
\end{aligned}
$$


In particular, if we take by $\psi(t)$ differentiable functions with compact support in $(0, T)$, we get

$$
(u(t), v)_{+}+\left(\left(\sum_{l=1}^{m} \tilde{a}_{l}(x) \mathfrak{D}_{l}+\delta a_{0}\right) u(t), v\right)_{L^{2}(\Omega)}+\frac{d}{d t}(u(t), v)_{L^{2}(\Omega)}=<f(t), v>
$$

in the sense of distributions. Now we have to show that $u(0)=u_{0}$. Indeed, multiplying (39) by $\psi(t)$ and integrating by parts we get

$$
\begin{aligned}
\int_{0}^{T}(u(t), v)_{+} \psi(t) d t & +\int_{0}^{T}\left(\left(\sum_{l=1}^{m} \tilde{a}_{l}(x) \mathfrak{D}_{l}+\delta a_{0}\right) u(t), v\right)_{L^{2}(\Omega)} \psi(t) d t- \\
& -\int_{0}^{T}(u(t), v)_{L^{2}(\Omega)} \psi^{\prime}(t) d t=(u(0), v)_{L^{2}(\Omega)} \psi(0)+\int_{0}^{T}<f(t), v>\psi(t) d t .
\end{aligned}
$$

Comparing it with (38) we get

$$
\left(u(0)-u_{0}, v\right)_{L^{2}(\Omega)} \psi(0)=0
$$

for all $v \in H^{+}(\Omega)$. Taking $\psi(0) \neq 0$ we receive $u(0)=u_{0}$.

The continuity follows from (15).

Corollary 5. Under the hypothesis of Theorem 2, the Problem 3 has one and only one solution $u(t) \in C\left(0, T ; L^{2}(\Omega)\right)$, if

$$
\mathfrak{R e}\left(\left(\left(\sum_{l=1}^{m} \tilde{a}_{l}(x) \mathfrak{D}_{l}+\delta a_{0}\right) v, v\right)_{L^{2}(\Omega)}\right) \geqslant 0
$$

for all $v \in L^{2}\left(0, T ; H^{+}(\Omega)\right)$.

Proof. The existence of the solution follows from the Theorem 4. Let us now show, that the solution is unique, if the condition (40) and the hypothesis of Theorem 4 are fulfilled. Indeed, let $v \in L^{2}\left(0, T ; H^{+}(\Omega)\right)$ is another solution of Problem 3. Denote by $w=u-v$. Then $w$ satisfies conditions of Problem 3 and

$$
(w, v)_{+}+\left(\left(\sum_{l=1}^{m} \tilde{a}_{l}(x) \mathfrak{D}_{l}+\delta a_{0}\right) w, v\right)_{L^{2}(\Omega)}+\frac{d}{d t}(w, v)_{L^{2}(\Omega)}=0
$$

for all $v \in H^{+}(\Omega)$, and $w(0)=0$. It follows from (13), that

$$
\frac{\partial w}{\partial t}+L w=0
$$

Multiplying scalar it by $w$ we have

$$
\|w\|_{+}^{2}+\left(\left(\sum_{l=1}^{m} \tilde{a}_{l}(x) \mathfrak{D}_{l}+\delta a_{0}\right) w, w\right)_{L^{2}(\Omega)}+\left(\frac{\partial w}{\partial t}, w\right)_{L^{2}(\Omega)}=0 .
$$

As the $\|w(t)\|_{+}^{2}$ is a real-valued function, therefore

$$
\|w\|_{+}^{2}+\mathfrak{R e}\left(\left(\frac{\partial w}{\partial t}, w\right)_{L^{2}(\Omega)}\right)+\mathfrak{R e}\left(\left(\left(\sum_{l=1}^{m} \tilde{a}_{l}(x) \mathfrak{D}_{l}+\delta a_{0}\right) w, w\right)_{L^{2}(\Omega)}\right)=0 .
$$


On the other hand,

$$
\mathfrak{R e}\left(\left(\frac{\partial w}{\partial t}, w\right)_{L^{2}(\Omega)}\right)=\frac{1}{2} \frac{d}{d t}\|w\|_{L^{2}(\Omega)}^{2} .
$$

It follows from (40), that

$$
2 \mathfrak{R e}\left(\left(\frac{\partial w}{\partial t}, w\right)_{L^{2}(\Omega)}\right)=\frac{d}{d t}\|w\|_{L^{2}(\Omega)}^{2} \leqslant 0
$$

and

$$
\|w(t)\|_{L^{2}(\Omega)}^{2} \leqslant\|w(0)\|_{L^{2}(\Omega)}^{2}=0,
$$

hence $w(t)=0$ for almost all $t \in[0, T]$, that completes the proof.

As we already mentioned, the embedding $H^{+}(\Omega)$ into $H^{1 / 2-\varepsilon}(\Omega)$ is rather sharp. Let us show, that the space $L^{2}\left(0, T ; H^{+}(\Omega)\right)$ can not be continuously embedded into $L^{2}\left(0, T ; H^{s}(\Omega)\right)$ for all $s>1 / 2$.

Eexample 6. Let $\Omega$ be a unit sphere in $\mathbb{C}$, matrix $\mathfrak{A}(x)$ has a form

$$
\mathfrak{A}(x)=\left(a_{i j}(x)\right)_{\substack{i=1,2 \\
j=1,2}}=\left(\begin{array}{cc}
1 & \sqrt{-1} \\
-\sqrt{-1} & 1
\end{array}\right)
$$

$S=\emptyset, a_{l}=0$ for $l=0,1, \ldots, m$, and $b_{1}=b_{0}=1$. Then the series

$$
u_{\varepsilon}(z, t)=\sum_{k=0}^{\infty} \frac{z^{k} t^{k / 2}}{T^{(k+1) / 2}(k+1)^{\varepsilon / 2}},
$$

$\varepsilon>0$, converges in $L^{2}\left(0, T ; H^{+}(\Omega)\right)$ and

$$
\left\|u_{\varepsilon}\right\|_{L^{2}\left(0, T ; H^{+}(\Omega)\right)}^{2}=\left\|u_{\varepsilon}\right\|_{L^{2}\left(0, T ; L^{2}(\mathbb{S})\right)}^{2}=2 \pi \sum_{k=0}^{\infty} \frac{1}{(k+1)^{1+\varepsilon}} .
$$

According to [20, Lemma 1.4]

$$
\left\|u_{\mathcal{\varepsilon}}\right\|_{L^{2}\left(0, T ; H^{s}(\mathbb{B})\right)}^{2} \geqslant \pi \sum_{k=0}^{\infty} \frac{k^{2 s-1}}{(k+1)^{1+\varepsilon}}, 0<s \leqslant 1 .
$$

It means, that for each $s \in(1 / 2,1)$ there exist $\varepsilon>0$ such that $u_{\varepsilon} \notin L^{2}\left(0, T ; H^{s}(\mathbb{B})\right)$. Hence, the space $L^{2}\left(0, T ; H^{+}(\mathbb{B})\right)$ can not be continuously embedded into $L^{2}\left(0, T ; H^{s}(\mathbb{B})\right)$ for all $s>1 / 2$.

The work was supported by the Foundation for the Advancement of Theoretical Physics and Mathematics "BASIS".

\section{References}

[1] O.A.Ladyzhenskaya, V.A.Solonnikov, N.N.Ural'tseva, Linear and Quasilinear Equations of Parabolic Type, Amer. Math. Soc., Providence, R.I., 1968

[2] J.L.Lions, E.Magenes, Non-Homogeneous Boundary Value Problems and Applications, Springer-Verlag, Berlin et al., 1972. 
[3] V.P.Mikhailov, Partial Differential Equations, Moscow, Mir, 1978.

[4] R.Temam, Navier-Stokes equations: Theory and Numerical Analysis, Studies in Math. and its Appl. 2, 1979.

[5] S.Agmon, A.Douglis, L.Nirenberg, Estimates near the boundary for solutions of elliptic partial differential equations satisfying general boundary conditions, Part 1. Comm. Pure Appl. Math., 12(1959), 623-727.

[6] S.Campanato, Sui problemi al contorno per sistemi di equazioni differenziale lineari del tipo dell'elasticitá, Ann. della Scuola Norm. Superiore, Cl. di Sci, Ser. III, 13(1959), no. 2, 223-258.

[7] S.Campanato, Proprietá di taluni spazi di distribuzioni e loro applicazione, Ann. della Scuola Norm. Superiore, Cl. di Sci, Ser. III, 14(1960), no. 4, 363-376.

[8] A.S.Peicheva, A.A.Shlapunov, On the completeness of root functions of Sturm-Liouville problems for the Lame system in weighted spaces. Z. Angew. Math. Mech., 95(2015), no. 11, $1202-1214$.

[9] A.N.Polkovnikov, A.A.Shlapunov, On spectral properties of a non-coercive mixed problem associated with the $\bar{\partial}$-operator, Journal of Siberian Federal University. Math. and Phys., 6(2013), no. $2,247-261$.

[10] A.Polkovnikov, A.Shapunov, On non-coercive mixed problems for parameter-dependent elliptic operators, Math. Commun., 15(2015), 1-20,

[11] A.Polkovnikov, A.A.Shlapunov, Sib. Math. J., 58(2017), no. 4, 676-686. DOI: $10.1134 /$ S0037446617040140

[12] A.Shlapunov, N. Tarkhanov, On completeness of root functions of Sturm-Liouville problems with discontinuous boundary operators, J. of Differential Equations, 10(2013), 3305-3337.

[13] J.J.Kohn, Subellipticity of the $\bar{\partial}$-Neumann problem on pseudoconvex domains: sufficient conditions, Acta Math., 142(1979), no. 1-2, 79-12.

[14] A.Shlapunov, N.Tarkhanov, Sib. Adv. Math., 26(2016), no. 1, 30-76.

DOI: $10.3103 / \mathrm{S} 105513441601003 \mathrm{X}$

[15] M.Schechter, Negative norms and boundary problems, Ann. Math., 72(1960), no. 3, 581-593. DOI: $10.1090 /$ S0002-9904-1960-10516-2

[16] H.Gaevsky, K.Greger, K.Zaharias, Nonlinear Operator Equations and Operator Differential Equations, Mir, Moscow, 1978.

[17] A.F.Filippov, Differential equations with discontinuous right-hand side, Nauka, Moscow, 1985 (in Russian).

[18] T.H.Gronwall, Note on the derivatives with respect to a parameter of the solutions of a system of differential equations, Ann. of Math., 20(1919), no. 2, 292-296.

[19] D.S.Mitrinović, J.E.Pečarić, A.M.Fink, Inequalities Involving Functions and Their Integrals and Derivatives, Mathematics and its Applications (East European Series), Vol. 53, Kluwer Ac. Publ., Dordrecht, 1991. 
[20] A.A.Shlapunov, Spectral decomposition of Green's integrals and existence of $W^{s, 2}$-solutions of matrix factorizations of the Laplace operator in a ball, Rend. Sem. Mat. Univ. Padova, 96(1996), 237-256.

\title{
О начально-краевой задаче для параболического дифференциального оператора с некоэрцитивными граничными условиями
}

\author{
Александр Н. Полковников \\ Сибирский федеральный университет \\ Красноярск, Российская Федерация
}

\begin{abstract}
Аннотация. Мы рассматриваем начально-краевую задачу для равномерно 2-параболического дифференциального оператора второго порядка в цилиндрической области в $\mathbb{R}^{n}$ с некоэрцитивными граничными условиями. В отличие от коэрцитивного случая в данной ситуации происходит потеря гладкости решения в пространствах соболевского типа. Пользуясь методом Галеркина, мы доказываем, что проблема имеет единственное решение в специальных пространствах Бохнера.
\end{abstract}

Ключевые слова: некоэрцитивная задача, параболическая задача, метод Галеркина. 